\title{
Heterogeneous geographic distribution of patients with aortic valve stenosis: arguments for new aetiological hypothesis
}

\author{
G Le Gal, V Bertault, E Bezon, J-C Cornily, J-A Barra, J-J Blanc
}

Heart 2005;91:247-249. doi: 10.1136/hrt.2004.037093

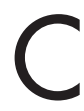
alcific aortic valve stenosis is by far the most common valve disease in developed countries. ${ }^{1}$ The first clinical symptoms usually appear in the late 60s. Prosthetic aortic valve replacement is the only effective treatment at present. ${ }^{2}$ The reasons for the occurrence of calcifications of the aortic cusps leading eventually after many years to aortic valve stenosis are unknown. Some risk factors, mainly those already recognised in vascular disease, have been found to be associated with aortic valve stenosis and are considered to cause the disease. ${ }^{3}{ }^{4}$ However, these factors are totally nonspecific and may be confounded with factors leading to other illnesses. Recently a genetic hypothesis has been proposed after observation of a few familial cases ${ }^{5}$ but remains largely challenged in the absence of gene determination. The aim of our study was to evaluate whether epidemiological data collected in a selected region of France could provide evidence in favour of this hypothesis.

\section{PATIENTS AND METHODS}

The list of all patients who had undergone aortic valve replacement for calcific aortic valve stenosis in the cardiac surgery department of the Brest University Hospital between January 1996 and June 2003 was obtained from the central computerised registration of medical diagnosis and surgical procedures of the hospital, coded according to the International classification of diseases, 10th revision. This list was compared, and if necessary completed, with the diary registries of all the surgical interventions performed in the cardiac surgery department.

For each patient, the following data were collected: name, sex, date of surgery, date of birth, and social security number. The social security number is a 15 digit number allowing identification for sex, year and month of birth, and geographic origin (region and commune). France is administratively divided into 22 provinces, about 100 regions, and 36000 communes. The commune is the smallest French administrative division. The canton is an intermediate division comprising four to eight communes. Only patients who where born in the region of Finistere were included in our analysis. The nature of the aortic valve disease was verified by examining every surgery report, which always describes the macroscopic aspect. When the surgeon mentioned that aortic valves were bicuspid the patient was excluded from analysis.

Our goals were, firstly, to establish the proportion of the population from each commune who had undergone aortic valve replacement for calcific aortic valve stenosis; secondly, to map the prevalence in the communes within the region to identify geographic clusters; and lastly, to detect significant differences between communes.

To assess the prevalence of the disease it was necessary to know the exact number of patients who had undergone aortic valve replacement originating from each commune during the study period. The choice of the reference population was an issue, as various options were possible. We could have used the number of inhabitants living in a commune at the time of the surgical intervention in the patients originating from this commune. This was not considered a good option, as it did not take into account major population movement in the preceding decades (urbanisation, second world war). Another possibility was to use the number of births in the commune during the year of the birth of each patient to obtain the incidence for each year in each commune. However, some little communes had only a few births in one year and experienced a few cases, leading to very variable results. Ideally, to assess more accurately the prevalence of surgical cases of aortic valve stenosis, the reference population could have been the number of people born in the commune during the same year as the patients and who were still alive at the time of the surgical interventions. These data are not available. We decided to use the population of the commune at the approximate date of birth of the patients and chose the 1926 census, which corresponds to the mean age (74 years) of patients who had undergone surgery between 1996 and 2003. These data were obtained from the 1926 census, which recorded 753801 inhabitants in the region. ${ }^{6}$

The results were expressed as the ratio of patients who had undergone aortic valve replacement for calcific aortic valve stenosis to 10000 inhabitants living in the commune in 1926. The relative risk was assessed as the risk of surgery in the commune as compared with the risk in the rest of the region. A 95\% confidence interval was estimated for each relative risk.

Visual study could not indicate the significance of observed clusters-that is, whether the geographic aggregations could have occurred by chance alone. Therefore, we used the method recommended by the Centers for Disease Control and Prevention. ${ }^{7}$ By this approach we assessed the deviation from chance expectation of the geographic pattern we observed as described by Ohno and colleagues. ${ }^{8}$ For this test, each commune was classified into one of three categories (0-9 cases, $10-19$ cases, and $\geqslant 20$ cases for each 10000 inhabitants living in 1926) and each pair of adjacent communes was identified. The statistical test analyses the number of adjacent concordant pairs-that is, the number of pairs of communes that were adjacent and had ratios in the same range. The expected number of adjacent concordant pairs is calculated by a simple formula given by Ohno and colleagues. ${ }^{8}$ The observed adjacent concordant pairs are compared with the expected number by a simple $\chi^{2}$ test.

\section{RESULTS}

During the study period 756 patients born in Finistere had undergone aortic valve replacement for calcific aortic valve stenosis in our institution. This gives a proportion of 10.03 patients in 10000 inhabitants living in 1926.

Figure 1 summarises the proportion of aortic valve replacement according to the commune of birth. In this map of 


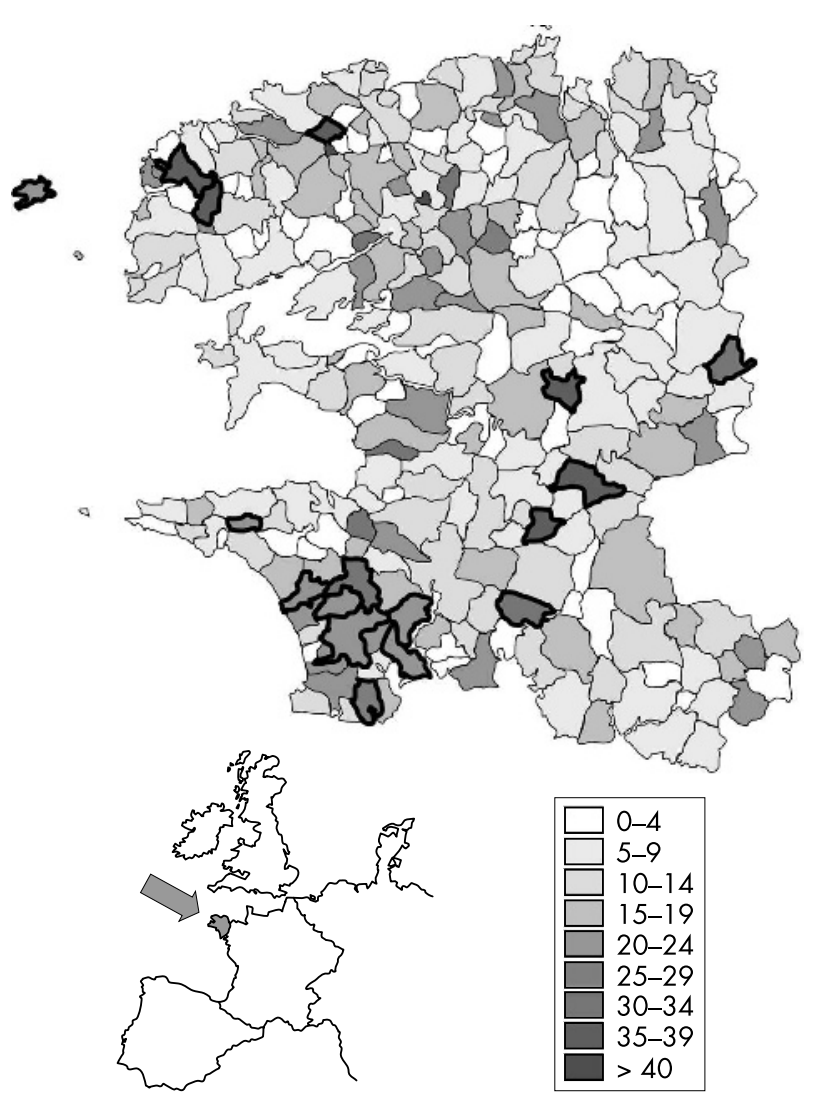

Figure 1 Map of Finistere, France. Ratios are the number of patients originating from each commune who underwent aortic valve replacement for aortic valve stenosis for each 10000 inhabitants living in the communes in 1926.

Finistere, each commune is shaded according to the proportion of cases. There is great heterogeneity among the communes, ranging from $0-52$ cases in 10000 inhabitants. It seems that there are several clusters of high proportion communes concentrated in a geographic area. This is more obvious when considering the highlighted communes, which are the communes that have a significantly higher proportion of cases-that is, a relative risk of surgery significantly greater than 1 .

Lastly, two areas are clearly prevalent: one in the southwest area of the region and the second in the northwest part.

After the division of the 283 communes into three ratio categories, we obtained 135 communes of low prevalence $(<10$ cases in 10000 inhabitants in 1926), 96 communes of intermediate prevalence (10-19 cases), and 48 of high prevalence $(\geqslant 20)$ (four islands were excluded). The total number of adjacent pairs in the full set of 283 communes was 712. The observed numbers of adjacent concordant pairs were 178,95 , and 32 within the low, intermediate, and high ratio category subsets, respectively. The expected numbers were 161,81 , and 20, respectively. The observed global number of adjacent concordant pairs was significantly higher than expected (305 $v 262, \mathrm{p}=0.009)$. In the high risk category, the observed number of adjacent concordant pairs was 32, as compared with 20 expected $(\mathrm{p}=0.008)$.

\section{DISCUSSION}

The main result of this study is evidence of a clear heterogeneous distribution of aortic valve replacement in the region of Finistere. The highly significant differences raised many issues.
Is Finistere an appropriate site for such a study? As fig 1 shows, Finistere is located in the western part of France at the extremity of the Brittany peninsula. It covers roughly $100 \times 100 \mathrm{~km}^{2}$ and is surrounded by the sea on three sides. This geographical situation explains the fact that inhabitants are a very static population. In particular, until the second world war, families remained in the same villages for generations. As an example, one of the authors has easily found that 10 generations of his own family lived in an area of less than $20 \mathrm{~km}$ from his own place of birth. Agriculture and fishing were the main economic activities with a homogeneous lifestyle all over the region. Lastly, the relatively dense population in the Finistere area (127 inhabitants $/ \mathrm{km}^{2}$ ) resulted in a sufficient number of patients. Furthermore, at the beginning of the 20th century, the population was equally distributed over the territory, and not concentrated in one or two big towns.

Are the collected data biased? The main goal of the study was to evaluate the geographic distribution of calcific aortic valve stenosis to support the hypothesis of genetic or environmental risk factors for this disease. A limitation of our study is that we analysed only the surgical cases. This may have selected a particular group of patients, potentially with a particular risk profile or with a particular pathological pattern. However, if selection of the surgical cases is not linked with geographic origin, this does not constitute a bias. Can the high incidence in some communes be explained by particular attention to this disease or by a particularly high density of physicians on these sites? This cannot be excluded. However, the density of general practitioners and cardiologists was not higher in these areas, and our region is in the range of what is reported for France.

Are the patients from these high risk zones more often referred to our institution than to others, leading to an overestimation of the proportion of cases? This is unlikely, as our hospital is the unique centre of cardiac surgery in the region of Finistere. Moreover, the two closest cardiac surgery centres are located 200 and $250 \mathrm{~km}$ from the border of our region and such an old population generally does not move to other places to undergo surgical intervention.

Lastly, we sought patients originating from Finistere who had undergone aortic valve replacement in these two cardiac surgery institutions. There were only a few patients (fewer than five a year in these two institutions). Therefore, it may be assumed that the reported results reflect a real difference in the incidence of this disease in some areas of the region.

Can these differences be explained by environmental or genetic factors? An environmental factor or a clustering of risk factors for arteriosclerosis ${ }^{3}$ may explain our results. However, the two areas with the highest proportion of cases are two agricultural zones, with roughly the same lifestyle. They are separated by almost $80 \mathrm{~km}$ and each of them is surrounded by important low risk zones. Moreover, our study is based on the place of birth rather than the place of residence. Indeed, although most people in our region live where they were born, our results are consistent with a genetic factor as well as environmental influences. The only way to show conclusively that calcific aortic stenosis is a genetic disease would be to identify a gene mutation in patients with the disease. To the best of our knowledge, this has never been done. In the absence of such a study our results provide a rationale to undertake or continue these investigations. Many difficulties have to be overcome to reach this goal. The next step may be to investigate one or more families in high risk areas with enough patients and enough controls.

In conclusion, this study reports that the distribution of geographic origin of patients undergoing aortic valve replacement for calcific aortic valve stenosis does not seem 
to be a random phenomenon. The geographical and historical particularities of the region of Finistere strengthen this conclusion and support new aetiological approaches to this disease.

\section{Authors' affiliations \\ G Le Gal, V Bertault, J-C Cornily, J-J Blanc, Department of Cardiology, Brest University Hospital, Brest, France \\ E Bezon, J-A Barra, Department of Cardiac Surgery, Brest University Hospital}

Correspondence to: Dr Grégoire Le Gal, Département de Cardiologie, Hôpital de la Cavale Blanche, Bd Tanguy Prigent, 29609 Brest cedex, France; gregoire.legal@chu-brest.fr

Accepted 24 March 2004

\section{REFERENCES}

1 Carabello BA. Aortic stenosis. N Engl J Med 2002;346:677-82.

2 Schwarz E, Baumann P, Manthey J, et al. The effect or aortic valve replacement on survival. Circulation 1982;66:1105-10.

3 Otto CM, Lind BK, Kitzman DW, et al. Association of aortic-valve stenosis with cardiovascular mortality and morbidity in the elderly. $N$ Engl J Med 1999;341:142-7.

4 Otto CM, Kuusisto J, Reichenbach DD, et al. Characterization of the early lesion of "degenerative" valvular aortic stenosis: histological and immunohistochemical studies. Circulation 1994;90:844-53.

5 Probst V, Schott JJ, Trochu JN, et al. Clinical evidence for hereditary factors in calcific aortic valvular stenosis in the elderly [abstract]. Circulation 2002;106(suppl):11639.

6 Charpy J. Dénombrements de la population des communes du Finistère (1790-1968). Bull Soc Arch Fin 1972;2:849-87.

7 Anon. Guidelines for investigating clusters of health events. MMWR Recomm Rep 1990;39(RR-11):1-23.

8 Ohno Y, Aoki K, Aoki N. A test of significance for geographic clusters of disease. Int J Epidemiol 1979;8:273-80.

\section{IMAGES IN CARDIOLOGY}

\section{Right to left shunt 20 years after sinus venous atrial septal defect closure}

A 29 year old woman with hypoxia of unknown aetiology, which was found one day after she underwent a knee joint operation, was admitted to our hospital. She had undergone an atrial septal defect (ASD) closure 20 years previously. Arterial $\mathrm{Po}_{2} \quad\left(\mathrm{PaO}_{2}\right)$ was $74 \mathrm{~mm} \mathrm{Hg}$ despite administration of $10 \mathrm{ml} / \mathrm{min}$ of oxygen $\left(\mathrm{PaO}_{2}\right.$ was $58 \mathrm{~mm} \mathrm{Hg}$ in room air). She had no symptoms and was not cyanotic. Chest $x$ ray was normal and there were no ECG abnormalities. There were no findings of pressure overload in the right atrium (RA) and right ventricle (RV) by transthoracic echocardiography (TTE). TTE revealed continuous abnormal flow signal, flowing into the left atrium (LA) on colour and pulsed Doppler, located just in the anterior side of the right pulmonary artery. Intravenous contrast (Levovist, Schering) injected from both upper limbs reached the LA and then the left ventricle (LV), with little contrast filling in the RA and RV. Intravenous contrast injected from the lower limb reached the RA normally (upper panel). Transoesophageal echocardiography showed distal superior vena cava (SVC) stenosis with SVC dilatation, and incomplete closure of sinus venous ASD. An SVC to LA (right to left) shunt flow signal was revealed by colour Doppler (lower panel). Mean pressures measured by using cardiac catheterisation were as follows: SVC $9 \mathrm{~mm} \mathrm{Hg}$, LA $5 \mathrm{~mm} \mathrm{Hg}$, RA $2 \mathrm{~mm} \mathrm{Hg}$.

As a result of distal SVC stenosis and residual ASD after inappropriate sinus venous ASD closure, SVC pressure exceeded LA pressure, and hence, SVC to LA shunt occurred through the residual ASD. This right to left shunt resulted in the patient's chronic hypoxia. Intravenous contrast echocardiography was useful for detecting that this patient had right to left shunting from the SVC to the LA, and transoesophageal echocardiography clearly revealed the complicated anatomical and physiological abnormality.

Fortunately, the patient had no history of systemic embolism for the preceding 20 years, or symptoms of hypoxia. After the successful corrective surgery, her hypoxia improved dramatically and she is doing well.

\section{N Watanabe \\ T Akasaka \\ K Yoshida non@med.kawasaki-m.ac.jp}

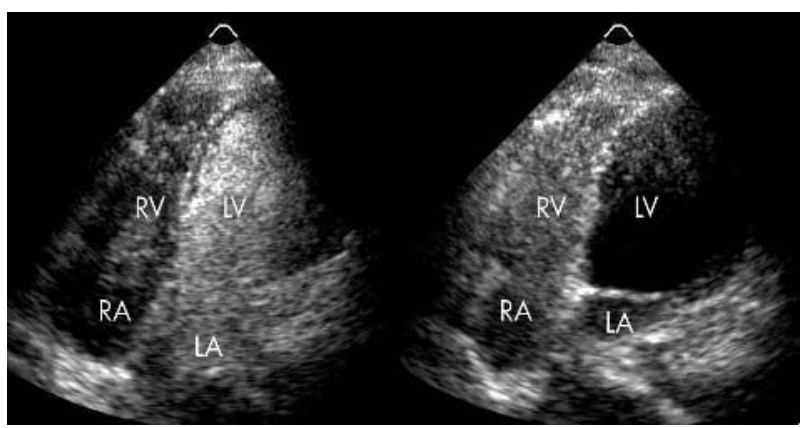

Transthoracic intravenous contrast echocardiographic images. Left panel: intravenous contrast injection from right upper limb. Contrast media reached the left side of the heart, with little contrast filling on the right side. Right panel: intravenous contrast injection from right lower limb. Contrast media reached the right side of the heart normally. LA, left atrium; LV, left ventricle; RA, right atrium; RV, right ventricle.

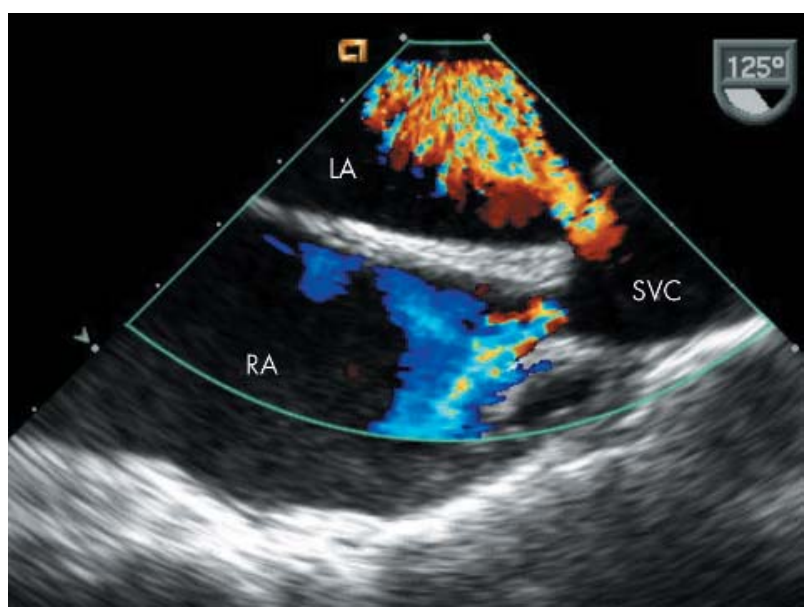

Transoesophageal echocardiographic image. A superior vena cava (SVC) to left atrium (LA) (right to left) shunt flow signal through the residual atrial septal defect is seen. The distal SVC is stenotic. 